\title{
DIRECT OBSERVATION OF GRAIN BOUNDARY DISLOCATION \\ CLIMB IN ION IRRADIATED GOLD BICRYSTALS
}

\author{
by \\ Y. Komem, P. Pétroff and R. W. Balluffi
}

21 December 1971

Cornel1 University

Ithaca, New York

Report \#1706

Issued by

The Materials Science Center

This report was prepared as an account of work sponsored by the United States Government. Neither the United States nor the United States Atomic Energy Commission, nor any of their employees, nor any of makes any warranty, subcontractors, or their employees, makes any warranty, express or implied, or assumes any legal liability or responsibility, for the accuracy, completeness or usefulness of any! information, apparatus, product or process disclosed, or represents that its use would not infringe privately owned rights. 


\section{DISCLAIMER}

This report was prepared as an account of work sponsored by an agency of the United States Government. Neither the United States Government nor any agency Thereof, nor any of their employees, makes any warranty, express or implied, or assumes any legal liability or responsibility for the accuracy, completeness, or usefulness of any information, apparatus, product, or process disclosed, or represents that its use would not infringe privately owned rights. Reference herein to any specific commercial product, process, or service by trade name, trademark, manufacturer, or otherwise does not necessarily constitute or imply its endorsement, recommendation, or favoring by the United States Government or any agency thereof. The views and opinions of authors expressed herein do not necessarily state or reflect those of the United States Government or any agency thereof. 


\section{DISCLAIMER}

Portions of this document may be illegible in electronic image products. Images are produced from the best available original document. 


\title{
DIRECT OBSERVATION OF GRAIN BOUNDARY DISLOCATION
}

CLIMB IN ION IRRADIATED GOLD BICRYSTALS ${ }^{+}$

\author{
by \\ Y. Komem ${ }^{++}$, P. Pêtroff ${ }^{*}$ and R. W. Balluffi ${ }^{* *}$
}

Thin-film gold bicrystals containing (001) low angle twist boundaries, with twist angles in the range $\theta=7-14^{\circ}$; and high angle twist boundaries, with $\theta \simeq 37^{\circ}$, were prepared by welding evaporated films together face-to-face. All the boundaries contained regular orthogonal arrays of intrinsic screw grain boundary dislocations (GBD's) as well as more randomly occurring extrinsic edge GBD's having Burgers vectors essentially perpendicular to the boundary. The specimens were irradiated on one face with $20 \mathrm{keV}$ gold ions producing excess self-interstitial atoms which diffused to the extrinsic GBD's causing them to climb in the boundary. The extrinsic GBD climb; in turn, caused the boundaries to act as overall. interstitial sinks. The climb motion was observed directly by transmission electron microscopy. The intrinsic screw GBD's in the high angle boundaries (and probably also in the low angle boundaries) remained unaffected during the process, but possibly

+ This work was supported by the U.S. Atomic Energy Commis sion under. Contract AT(11-1)-3158. Additional suppor.t. was received from the Advanced Research Projects Agency through the technical facilities of the Materials Science Center at Cornell University

++. Now at the Technion-Israel Institute of Technology, Haifa, Israe 1.

* Now at Bell Telephone Laboratories, Murray Hill, New Jersey, 07974.

* Department of Materials Science and Engineering, Cornell University, Ithaca, New York, 14850. 
they acted as short circuiting pipes which delivered interstitials to the extrinsic GBD's. It is concluded generally that both low and high angle boundaries may act as point defect sinks (sources), by means of the climb of GBD's having Burgers vectors with components normal to the boundary plane. This process is actualiy a form of internal crystal growth (dissolution) with the GBD's acting as "internal crystal ledges". 


\section{INTRODUCTION}

The ability of grain boundaries to act as sources (or sinks) for point defects has been demonstrated on many occasions (for example: Balluffi and Seigle 1955, Alexander and Balluffi 1957, Norris 1971). Recently, it has been suggested that the climb of suitable grain boundary dislocations (GBD's) must be responsible for the point defect source (or sink) action of grain boundaries (Gleiter 1969a,b, Schober and Balluffi 1971b).

In the present work we demonstrate the essential role played by GBD climb in allowing both low and high angle twist boundaries to act as sinks for excess self-interstitial atoms (interstitials). Thin-film bicrystal gold specimens possessing grain boundaries containing edge GBD's with Burgers vectors perpendicular. to the boundary plane were bombarded on one face with $20 \mathrm{keV}$ gold ions. This irradiation produced a current of excess interstitials which diffused to the GBD's causing them to climb. The GBD climb, in turn, caused the boundaries to act as overall sinks. The GBD climb motion was observed directly by transmission electron microscopy.

\section{EXPERIMENTAL}

\subsection{Specimen Geometry}

The gold bicrystal specimens, containing pure (0ii) twist boundaries, were prepared by welding (001) evaporated single.crystal films together face-to-face using a technique described previousty (Schober and Balluffi 1969, 1970). Ex- 
tensive grain boundary areas were prepared by this technique which could be readily examined at normal incidence in the electron microscope.

Previous work (Schober and Balluffi, 1970, 1971a) has shown that $(001)$ twist boundaries prepared by this method contain the following two types of GBD's:

(1) Intrinsic screw GBD's. These GBD's are present in regular orthogonal. networks and are part of the equilibrium boundary structure. So far, these arrays have been observed. only in ranges of misorientation centered around singular misorientations corresponding to a high density of coincidence sites in the unrelaxed boundary. The role of these GBD's is to restore most of the boundary area to the relatively good fit characteristic of the singular misorientation. The most familiar example of such an intrinsic GBD structure is the classical low angle boundary consisting of a network of orthogonal Screw GBD's possessing Burgers vectors $\vec{b}=a / 2<110\rangle$. In this case the singular misorientation corresponds to zero twist angle with all atoms across the boundary in coincidence (i.e., $\Sigma=1$, where $\Sigma$ is the reciprocal of the fraction of atoms in coincidence). Similar intrinsic GBD networks have been detected near the singular twist angles $\theta_{s}=22.6^{\circ}(\Sigma=13), 28.1^{\circ}(\Sigma=17)$. and $36.9^{\circ}(\Sigma=5)$. Even though such GBD's have not yet been detected over the entire range of possible twist misorientations, we believe that they exist over most, if not all, of the total range (Balluffi, Komem and Schober, 1971).

(2) Extrinsic GBD's. These GBD's are extra dislocations 
which happen to be present in the boundary and which are not part of the equilibrium structure. They are generally distributed in a rather disorganized fashion, and their strain fields do not cancel at large distances. These GBD's have been found to be essentially edge dislocations with their Burgers vectors perpendicular to the boundary. The most likely sources of such GBD's in the present specimens were lattice dislocations which entered the boundaries during plastic deformation during the welding operation, or else (002) atomic steps which were present on the surfaces of the evaporated films prior to welding (Schober and Balluffi, 1971a).

In the present work two types of boundaries were investigated: $\quad$ i.e., low angle $\Sigma=1 \quad\left(\theta_{s}=0\right)$ boundaries having twist angles $\theta$, in the range $\theta=7$ to $14^{\circ}$ and high angle $\sum=5 \quad\left(\theta_{s}=36.9^{\circ}\right)$ boundaries having twist angles. within a few degrees of $37^{\circ}$.

Examples of the initial appearance of the GBD struc-: tures in these boundaries are given in figs. 1a, 2a and $3 a$. Here, the extrinsic GBD's appear as wide images exhibiting either symmetric "black-white-black" (BWB) or "whité-blackwhite" (WBW) contrast. This contrast was produced using a diffraction vector, $\vec{g}$, lying in the boundary plane so that $\vec{g} \cdot \vec{b}=0$, and $\vec{g} \cdot \vec{b} \times \vec{u} \neq 0$ where $\vec{b}$. is the Burgers vector and $\vec{u}$ is the dislocation line tangent vector. A full discussion of this type of contrast, has been given elsewhere (Balluffi, Woolhouse and Komem, 1971). The grids of intrinsic GBD's were generally observed as part of the background structure of the high angle $\Sigma=5$ boundaries as seen in figs. $2 \mathrm{a}$ and $2 \mathrm{~b}$. The grid spacing, 
d, varies inversely with the deviation from the singular twist angle $\theta_{s}=36.99^{\circ}$ and is given by

$$
d=\frac{\vec{b}}{\left|\theta_{s}-\theta\right|} .
$$

The corresponding intrinsic. GBD grids in the low angle $\Sigma=1$ boundaries were not clearly observable in most cases since their spacings, according to eqn. (1), were in the range $12-$ $24 \AA$.

A detailed arialysis of the manner in which the extrinsic and intrinsic GBD's interact has been described elsewhere (Schober and Balluffi, 1971a) and is illustrated in fig. 4. If we imagine that an extra dislocation with the lattice Burgers vector $\vec{b}=a / 2[10 \overline{1}]$ becomes imbedded in an initially uniform grid of intrinsic screw GBD's in either a $\Sigma=1$ or $\Sigma=5$ boundary, the structures shown in fig. 4 result when the dislocation lies at either 0 or $45^{\circ}$ with respect to one set of the intrinsic GBD's. Various interactions occur which produce the complex line structures shown along $A B$ and $C D$. The Burgers vectors of the different segments which are involved are as follows:

$$
\Sigma=1 \text { boundary }\left\{\begin{array}{l}
\vec{b}_{1}=a / 2[\overline{1} \overline{1} 0] \\
\vec{b}_{2}=a / 2[\overline{1} 10] \\
\vec{b}_{3}=a / 2[0 \overline{1}] \\
\vec{b}_{4}=a / 2[01 \overline{1}] \\
\vec{b}_{5}=a / 2[\overline{1} 0 \overline{1}]
\end{array}\right.
$$

\footnotetext{
* The position of the $i^{\text {th }}$ segment corresponding to each $\vec{b}_{i}$ is
indicated in fig. 4.
} 


$$
\sum=5 \text { boundary }\left\{\begin{array}{l}
\vec{b}_{1}=a / 10[\overline{1} 30] \\
\vec{b}_{2}=a / 10[310] \\
\vec{b}_{3}=a / 10[\overline{2} 15] \\
\vec{b}_{4}=a / 10[2 \overline{1} 5] \\
\vec{b}_{5}=a / 10[125]
\end{array}\right.
$$

In addition, it is readily shown (Schober and Balluffi, 1971a) that the effective Burgers vectors of the line defects along $A B$ and $C D$ are just $a / 2[00 \overline{1}]$ when $p=1.5 \mathrm{~d}$ in fig. $4^{*}$. (See distance $p$ in fig. 4.) These defects are therefore effective edge dislocations (of rather complicated structure) with effective Burgers vectors normal to the boundary plane.

\subsection{Irradiation and Microscopy}

Prior to irradiation, the specimens were mounted in electron microscope specimen holders, and four or five typical grain boundary areas were selected and photographed in the microscope. Dark field images of the GBD structure in each area were obtained using a strong two-beam condition exclusively in the crystal facing upwards in the microscope and also in the crystal facing downwards (see below). Extensive discussion of the GBD contrast to be expected under these conditions has been given elsewhere (Balluffi, Woolhouse and Komem, 1971).

The specimens, while still clamped in the electron microscope cartridge, were then transferred to a heavy ion accelerator. (Scanlan, Styris and Seidman, 1971) and were bombarded at room temperature on one face with $20 \mathrm{keV}$ gold ions as

We note that this result shows that the horizontal component of the original extra dislocation has been eliminated exactly by interactions with the intrinsic GBD network. 
shown schematically in fig. 5. The specimen was oriented with the [001] direction (i.e., surface normal) about $15^{\circ}$ away from the incident beam direction in order to minimize channeling effects. We note that it was found experimentally that less microscope contamination formed on the face of the specimen which was facing downwards in the electron microscope, and the specimens were therefore irradiated on this face. Crystal 1 , which was the crystal facing away from the ion beam (fig. 5), was therefore always the upper crystal during electron microscope observation.

Previous work (Thomas, Schober and Balluffi, 1969)

has shown that under such bombardment conditions the $20 \mathrm{keV}$ ions are stopped in displacement cascades in a region well within $\sim 200 \AA$ of the surface, and that vacancy-rich depleted zones are produced in the cascades. The depleted zones form as a result of the ejection from the cascade regions of a net number of interstitials by means of long-range focused replacement colIision sequences (Beavan, Scanlan and Seidman, 1971; Pétroff and Seidman, 1971,1972$)$. The net result in the present specimens is the deposition of interstitials well within crystal 2 with the vacancy defects left behind essentially at the surface as indicated. schematically in fig. 5. Since the interstitials are certainly mobile at room temperature (Schilling, Burger, Isebeck and Wenzl, 1970) this experimental arrangement exposed the grain boundary to a flux of excess mobile interstitials.

After irradiation, the specimens were again placed 
in the microscope, and the previously photographed areas were again located and photographed. A substantial fraction of the depleted zones caused by $20 \mathrm{keV}$ ions is known to be visible in the electron microscope in the form of dislocation loops or "black spot defects" (Thomas, Schober and Balluffi, 1969; Merkle, Singer and Wrobel, 1970), and these were readily visible in the present work when the two-beam diffraction condition was established exclusively in the lower crystal (Crystal 2) as seen in fig. 6b. These effects generally obscured the structure of the boundary. However, when the corresponding diffraction condition was established in the upper crystal (Crystal 1) none of the vacancy defects were imaged, as expected, and the grain boundary structure could be observed clearly as seen in fig. 6a. All of our grain boundary observations after irradiation were therefore made with only the upper crystal in a strong two-beam diffraction condition.

\section{RESULTS}

The following results were obtained:

\subsection{High Angle $(\Sigma=5)$ Boundaries}

3.1.1. The extrinsic GBD!s which were widely spaced in the boundary (i.e., $>1000 \AA$ ) climbed over large distances in the boundary plane as a result of the irradiation as seen in figs. 2 and 3. The average climb distance, $\bar{w}$, was defined as

$$
\bar{W} \equiv \frac{A}{L_{0}},
$$

where $A$ is the area swept out by the climbing GBD, and $L_{0}$ is

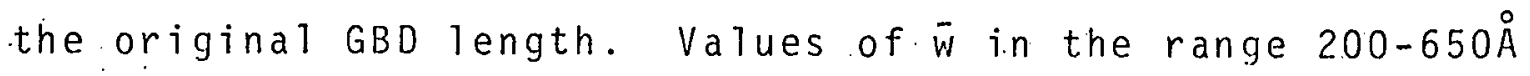


were obtained for ion doses in the range $\phi=2$ to $4 \times 10^{13^{3}}$ ions $\mathrm{cm}^{-2}$.

3.1.2. When the spacing of the extrinsic GBD's was less than $\sim 200 \AA$, the climb distance became small and difficult to measure with any accuracy.

3.1.3. The direction of the climb of the extrinsic GBD's was correlated with the BWB or WBW nature of the diffraction contrast calculated previously for such edge dislocations (Ba) $\overrightarrow{1}$ uffi, Woolhouse and Komem, 1971), and it was verified that the climb occurred by the absorption of interstitials rather than vacancies; $i . e$. , the climb always occurred in a. direction away from the position of the "extra plane" as determined from the nature of the contrast. A case where GBD's with opposite Burgers vectors. (as revealed by a reversal of the sign of their triple contrast) climbed in opposite directions is seen at $B$ and $C$ in $f i g .3$.

3.1.4. The climb distance of the widely spaced (i.e., s $1000 \AA$ ) extrinsic GBD's decreased monotonically with an increase in the intrinsic GBD spacing (fig. 7).

3.1.5. In areas free of extrinsic GBD's the intrinsic GBD's remained unaffected.

3.1.6. The regular arrangement of the intrinsic GBD network was disturbed in regions which were swept out by the climbing. extrinsic GBD's.

\subsection{Low Angle $(\Sigma=1)$ Boundaries}

3.2.1. The extrinsic GBD's climbed over large distances in the boundary plane (fig. 1). Values of $\vec{w}$ in the range $100-300 \AA$ 
were obtained for $\phi=2$ to $4 \times 10^{13}$ jons $\mathrm{cm}^{-2}$.

3.2.2. The extrinsic GBD's tended to adopt kinked configurations during climb with individual segments oriented along $<110>$ directions (fig. 1).

3.2.3. No observations of the behavior or influence of the intrinsic GBD's were made, since these GBD's were generally too closely spaced to be observed conveniently.

\section{DISCUSSION}

\subsection{High Angle $(\Sigma=5)$ Boundaries}

The present results show directly that the high angle twist boundaries acted as sinks for the mobile interstitials as a result of the climb of the extrinsic dislocations*. The extrinsic GBD climb added atoms to the lattice structure in the grain boundary region thereby causing the two crystals to move apart. The grid of intrinsic screw GBD's remained unaffected lexcept in the direct vicinity of the climbing extrinsic GBD's) and evidently did not act as interstitial sinks. This behavior is to be expected, since a. screw dislocation can only absorb point defects by increasing its projected area on a plane perpendicular to its length. Since the Burgers vectors of the intrinsic screw GBD's are not lattice vectors, it was topologically impossible for them to absorb point de = fects by climb.

Previous work by Schober and Balluffi (1971b), and additional control experiments in the present work, proved that the observed motion of the extrinsic GBD's couid not have been due to thermal or mechanical effects during handling but could only have been due to interstitial-induced climb. 
All of the observed results appear to be consistent with a model in which the extrinsic GBD's acted as relatively efficient sinks for the mobile interstitials. For example, when the extrinsic GBD's were closer than about $200 \AA$ their diffusion fields evidently overlapped, and their climb distances decreased correspondingly.

The detailed manner by which the complex extrinsic GBD line structures shown in fig. 4 translated by climb is. not entirely:clear. The various segments 1 abelled 3,4 and 5 (fig. 4) all have strong edge character and would be expected to climb approximately in the manner of edge dislocations (Balluffi, 1969). In the general case when the extrinsic GBD was not parallel to one of the intrinsic GBD sets (e.g., $A B$ in fig. 4) continuous transiation of the line would require considerable distortions of the regular arrangement shown in fig. 4 to occur periodically during the motion. Such distortions were undoubtedly responsible for the disturbed regions in the intrinsic GBD network found in the wake of the climbing extrinsic GBD's .

The observed increase in extrinsic GBD climb distance with decreasing intrinsic GBD spacing is of considerable interest and could conceivably. be due to either:

(a) increased transport of interstitials to the climbing extrinsic GBD's by short-circuiting along the increased number of intrinsic GBD's which acted as fast divergenceless transmission pipes.

(b) an increased climb rate due to the increased 
number of nodes in the extrinsic GBD configuration (fig. 4) which acted as heterogeneous sites for jog nucleation (Balluffi, 1969). Unfortunately, it does not seem possible to distinguish between these possibilities on the basis of our limited knowledge of the present system.

Finally, we demonstrate by means of a simple orderof-magnitude estimate that the observed climb distances are not inconsistent with the previous model and discussion. The number of interstitials deposited within the specimen per unit area of surface may be estimated as

$$
N \simeq \frac{f \emptyset E}{2 E_{d}}=10^{15} \mathrm{~cm}^{-2},
$$

where,

$$
\begin{aligned}
\emptyset= & \text { dose }=3 \times 10^{13} \text { ions } \mathrm{cm}^{-2} \\
E= & \text { incident ion energy }=2 \times 10^{4} \mathrm{eV} \\
E_{d}= & \text { displacement energy }=35 \mathrm{eV} \text { (Wollenberger, 1970) } \\
f= & \text { fraction of interstitials which survived their cas }- \\
& \text { cade and were ejected in the forward direction } \simeq \frac{3}{4} .
\end{aligned}
$$

Here, we have employed the model of Kinchin and Pease (1955) in order to estimate the number of interstitials produced. The value $f \simeq \frac{1}{4}$ is not inconsistent with current ideas about interstitial survival and also available field ion microscope observations of interstitial distributions around cascade regions in tungsten and platinum (Beavan, Scan'an and Seidman, 1971; Petroff and Seidman, 1971, 1972). The extrinsic GBD climb dis- 
tance is then

$$
\bar{w} \simeq \frac{\mathrm{m} \mathrm{Na} \mathrm{Na}^{2} \mathrm{D}}{2}=300 \AA \text {, }
$$

where,

$$
\begin{aligned}
a= & \text { lattice parameter }=4.08 \AA \\
D= & \text { average extrinsic } G B D \text { spacing } \simeq 1500 \AA \\
m= & \text { fraction of deposited interstitials which reached } \\
& \text { the extrinsic GBD's } \simeq \frac{1}{4} .
\end{aligned}
$$

The value of $m$ is, of course, difficult to estimate, since the mobile interstitials may: 1) recombine with vacancy defects; 2) diffuse to grain boundary voids; 3) diffuse to the front surface; 4) diffuse to the back surface; 5) diffuse to the extrinsic GBD's by means of short-circuiting along the intrinsic GBD network or along the singuiar $(\Sigma=5)$ interface; 6$)$ diffuse directiy to the extrinsic GBD's. In addition, we do not have much reliable information about the range of the focused replacement collision sequences*, the short circuiting behavior of the intrinsic GBD network or the singular interface, the line sink efficiency of the extrinsic GBD's, or the diffusive characteristics of the mobile interstitials. Nevertheless, the value of $\bar{w} \simeq 300 \AA$ estimated on the basis of $m \simeq \frac{3}{4}$ is close to the observed values, and we conclude that our previously developed picture of the climb process is not inconsistent with the observed climb distances.

Venables and Thomas (1970) argue that the range at temperatures well below $25^{\circ} \mathrm{C}$ must be in the neighborhood of $2100 \AA$, whereas Seeger (1970) claims that it must be $\sim 1000 \AA$. 


\subsection{Low Angle $(\Sigma=1)$ Boundaries}

Considerably less information was obtained regarding the geometry of the climb processes in the $\Sigma=1$ boundaries, since the intrinsic GBD networks were not resolved clearly. However. the magnitudes of the observed climb distances may again be understood on the basis of the estimate given above for the $\Sigma=5$ case. Again, it seems likely that large scale climb of the intrinsic screw GBD's did not occur, since in this case such climb would have produced a large interaction energy between these closely spaced and parallel dislocations. The tendency to form extrinsic GBD segments along <110> directions was evidently due to a marked anisotropy of the line tension in the boundary plane. This conclusion is supported by numerous other observations in our 7 aboratory which have shown that extrinsic GBD's in annealed $\Sigma=1$ boundaries tend to lie in segments parallel to $\langle 110\rangle$ directions.

\section{CONCLUSION}

We may conclude, in general, that both low and high angle boundaries may act as point defect sinks (sources) by means of the climb in the boundary plane of GBD's having Burgers vectors with components normal to the boundary plane.

This process is actually a form of internal crystal growth (dissolution) with the GBD's acting as "internal crystal ledges".

\section{ACKNOWLEDGEMENT}

The authors wish to thank Dr. J. Robinson and Professor D. N. Seidman for valuable discussions. 


\section{REFERENCES}

Alexander, B. H., and Balluffi, R. W., 1957, Acta Met., $\underline{5}$, 666 .

Balluffi, R. W., 1969, Phys: Stat. Sol., 31, 443.

Balluffi, R. W., Komem, Y., and Schober, T., 1971, Materials Science Center Report 1606 , Cornel1 University, Ithaca, New York.

Balluffi, R. W., and Seigle, L. L., 1955, Acta Met., 3, 170.

Balluffi, R. W., Woolhouse, G. R., and Komem, Y., 1972, Materials Science Center Report 1659, Cornel1 University, Ithaca, New York.

Beavan, L. A., Scanlan, R. M., and Seidman, D. N., 1971, Materials Science Center Report 1516, Cornell University, I thaca, New York.

Gleiter, H., 1969a, Acta Met., 17, 565; 1969b, Ibid., 17, 853.

Kinchin, G. H., and Pease, R. S., 1955, Reports on Prog. in Phys., No. 18, 1 .

Merkle, K. L., Singer, L. R., and Wrobel, J. R., 1970, Appl. Phys. Lett., 17, 6 .

Norris, D. I. R., 1971, Phil. Mag., 23, 135.

Pétroff, P., and Seidman, D. N., 1971, Appl. Phys. Lett., 18, $518 ; 1972$, to be published.

Scanlan, R. M., Styris, D. L., and Seidman, D. N., 1971, Phil. Mag., 23, 1439 .

Schilling, W., Burger, G., Isebeck, K., and Wenzl, H., 1970, Proc. Intern. Conf. on Vacancies and Interstitials in Metals, Jülich (North-Holland Publishing Co., Amsterdam), p. 255 .

Schober, T., and Balluffi, R. W., 1969, Phil. Mag., 20, 511; 1970, Phil Mag., 21, 109; 1971a, Phil. Mag., 24, 165; $1971 \mathrm{~b}$, Phil. Mag., 24, 469 .

Seeger, A., 1970, Rad. Effects, 2, 165.

Thomas, L. E., Schober, T., and Balluffi, R. W., 1969, Rad. Effects, $1,257$.

Venables, J. A., and Thomas G. J., 1970, Proc. Intern. Conf. on Vacancies and Interstitials in Metals, Jülich (NorthHolland Publishing Co:, Amsterdam), p. 531. 
Wollenberger, H., 1970, Proc. Intern. Conf. on Vacancies and Interstitials in Metals, Jiilich (North-Holland Publishing (o., Amsterdam), p. 215. 


\section{FIGURE CAPTIONS}

Fig. 1: Low angle $(\Sigma=1)$ twist boundary. $\theta \simeq 14^{\circ}$.

(a) GBD structure before irradiation. (b), (c) and

(d) Same area after successive irradiations with $20 \mathrm{keV}$ gold ions to final dose of 2 to $4 \times 10^{13}$ ions $\mathrm{cm}^{-2}$. All are dark field images using $\vec{g}=(200)_{1}$. Note climb of extrinsic GBD segments at, for example, $A$ and $B$. Circular and oval-shaped regions are voids in the grain boundary resulting from the welding process.

Fig. 2: High angle $(\Sigma=5)$ twist boundary. $\theta \simeq 37^{\circ}$.
(a) GBD structure before irradiation.
(b) Same area

as (a) after irradiation with $20 \mathrm{keV}$ gold ions to a dose of 2 to $4 \times 10^{13}$ ions $\mathrm{cm}^{-2}$. Both are dark field images using $\vec{g}=(200)_{1}$. (c) Schematic drawing showing climb of the extrinsic GBD's at $A, B$ and $C$.

Fig. 3: High angle $(\Sigma=5)$ twist boundary. $\theta \simeq 37^{\circ}$.

(a) GBD structure before irradiation. (b) Same area as (a) after irradiation with $20 \mathrm{keV}$ gold ions to a dose of 2 to $4 \times 10^{13}$ ions $\mathrm{cm}^{-2}$. Both are dark field images using $\vec{g}=(200)_{1}$. (c) Schematic drawing showing climb of the extrinsic GBD's at $A, B$ and $C$.

Fig. 4: Schematic diagram of extrinsic GBD structures along $A B$ and $C D$. Values of the subscript $i$ in the expression $\vec{b}_{i}$ for the Burgers vector are indicated for the dislocation segments bounding several cells of the network [see eqns. (2) and (3)]. 
Fig. 5: Schematic diagram of the damage distribution in the ion irradiated bicrystals. The arrows leaving the depleted zones near. the surface represent the paths of the long-range focused replacement sequences.

Fig. 6: High angle $(\Sigma=5)$ boundary after irradiation with $20 \mathrm{keV}$ gold ions to a dose of 2 to $4 \times 10^{13}$ ions $\mathrm{cm}^{-2}$. $\theta \simeq 37^{\circ}$. (a) Dark field image using $\vec{g}=(200)_{1}$. Dark field image using $\vec{g}=(200)_{2}$.

Fig. 7: Average extrinsic GBD climb distance, $\bar{w}$, as a function of the intrinsic GBD network spacing, $d$, for high angle $(\Sigma=5)$ twist boundaries irradiated to doses of 2 to $4 \times 10^{13}$ ions $\mathrm{cm}^{-2}$. Extrinsic GBD spacings $>1000 \AA$. 

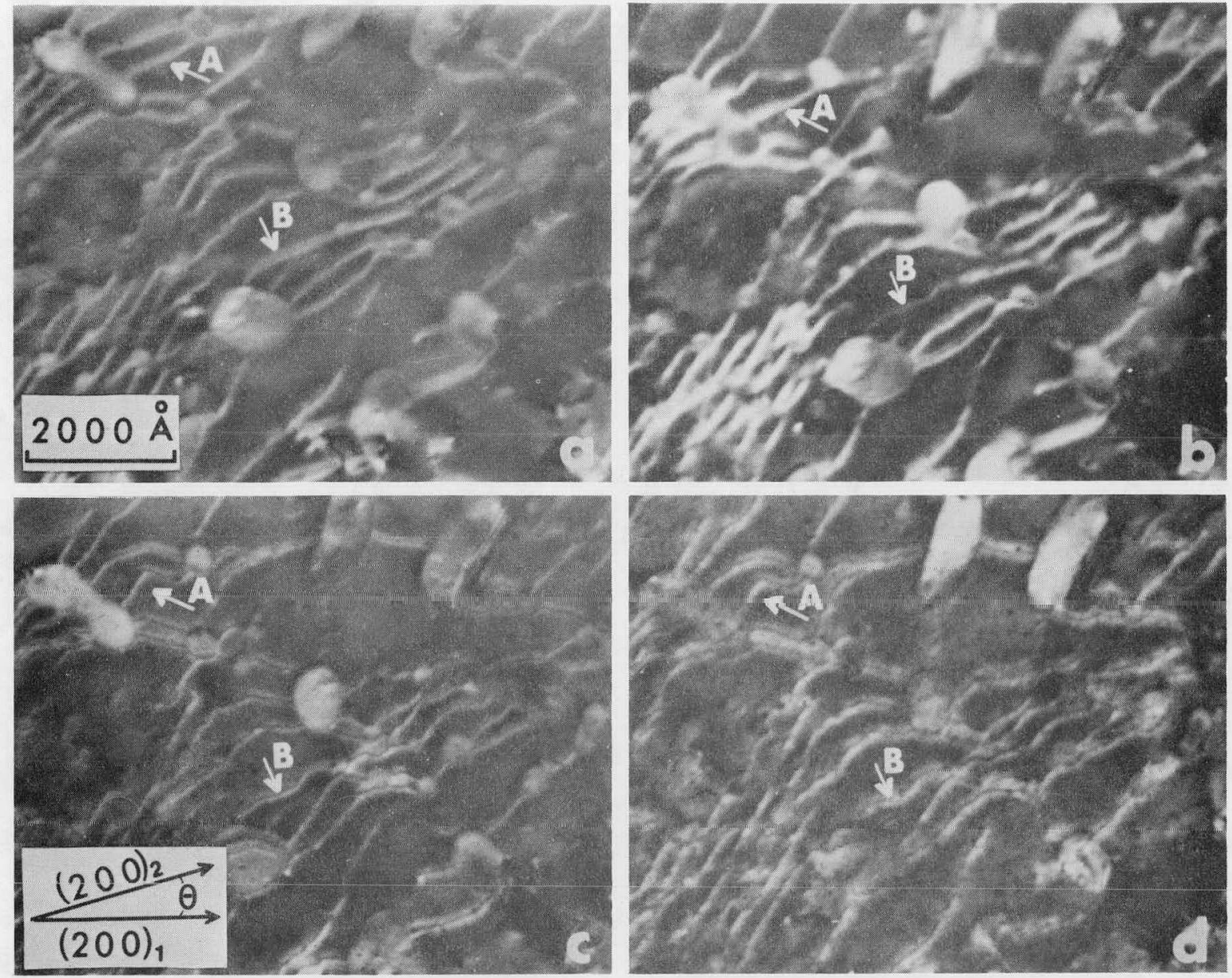

Figure 1. 

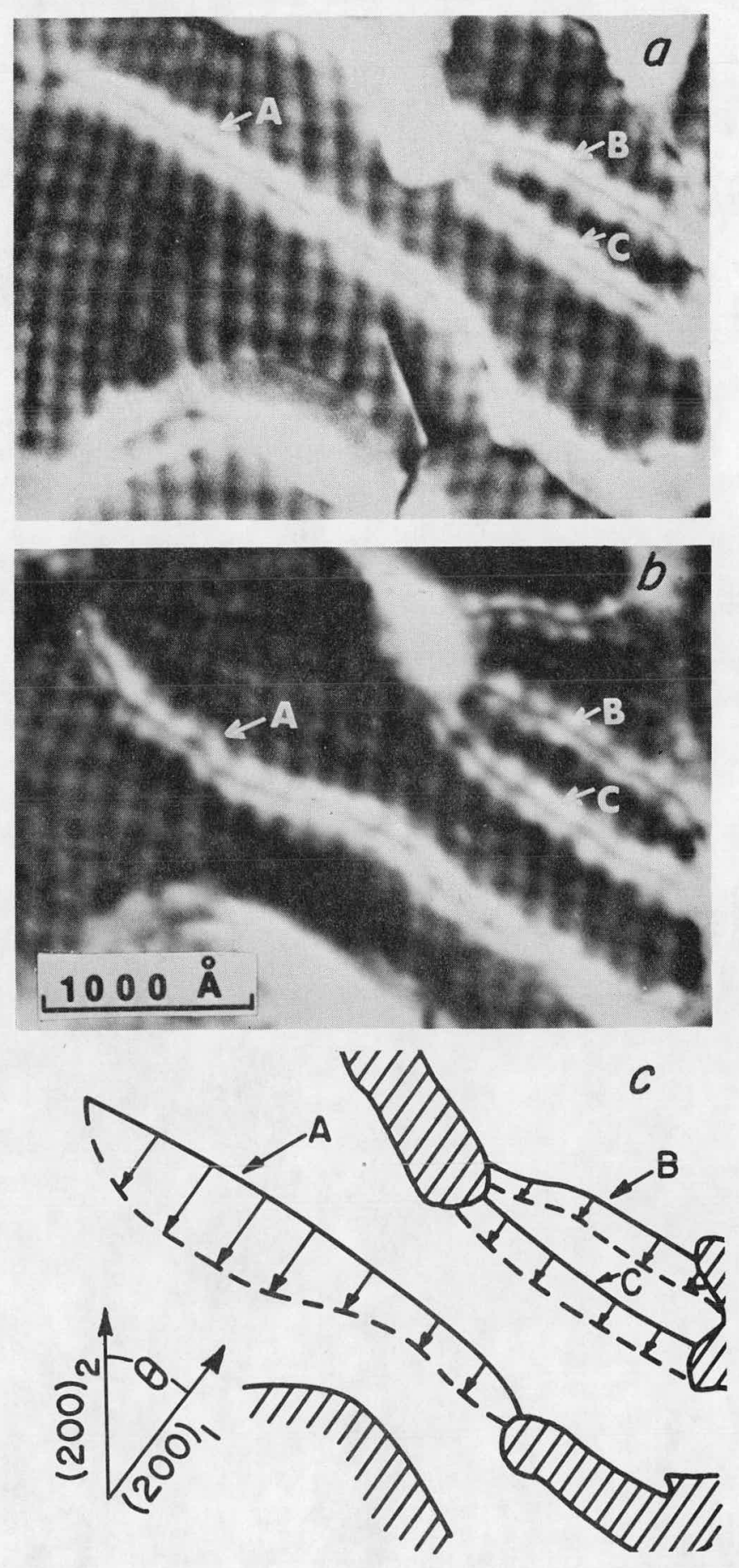

Figure 2. 

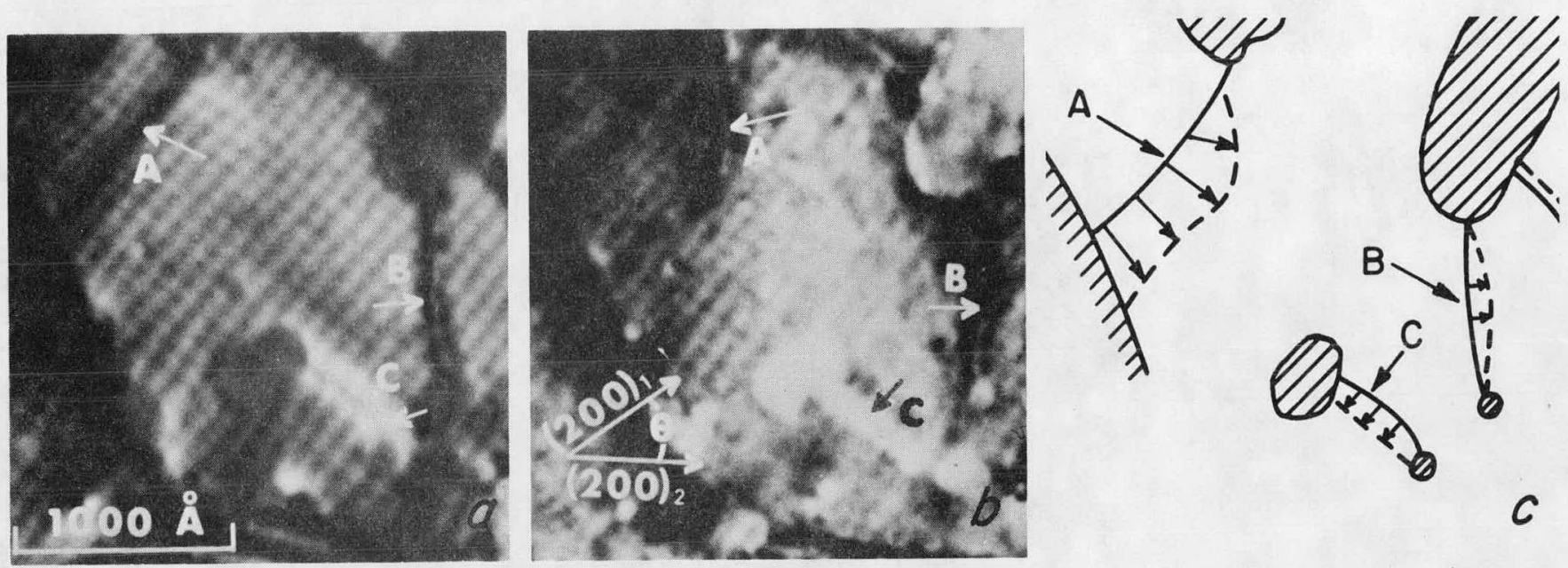

Figure 3. 


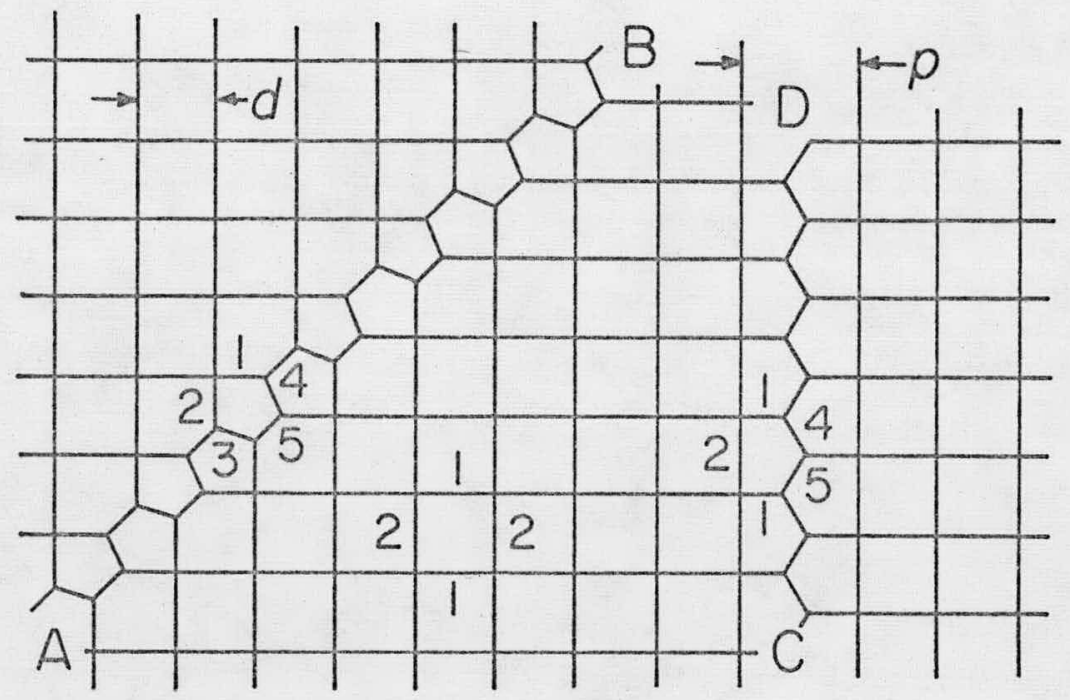

Figure 4. 


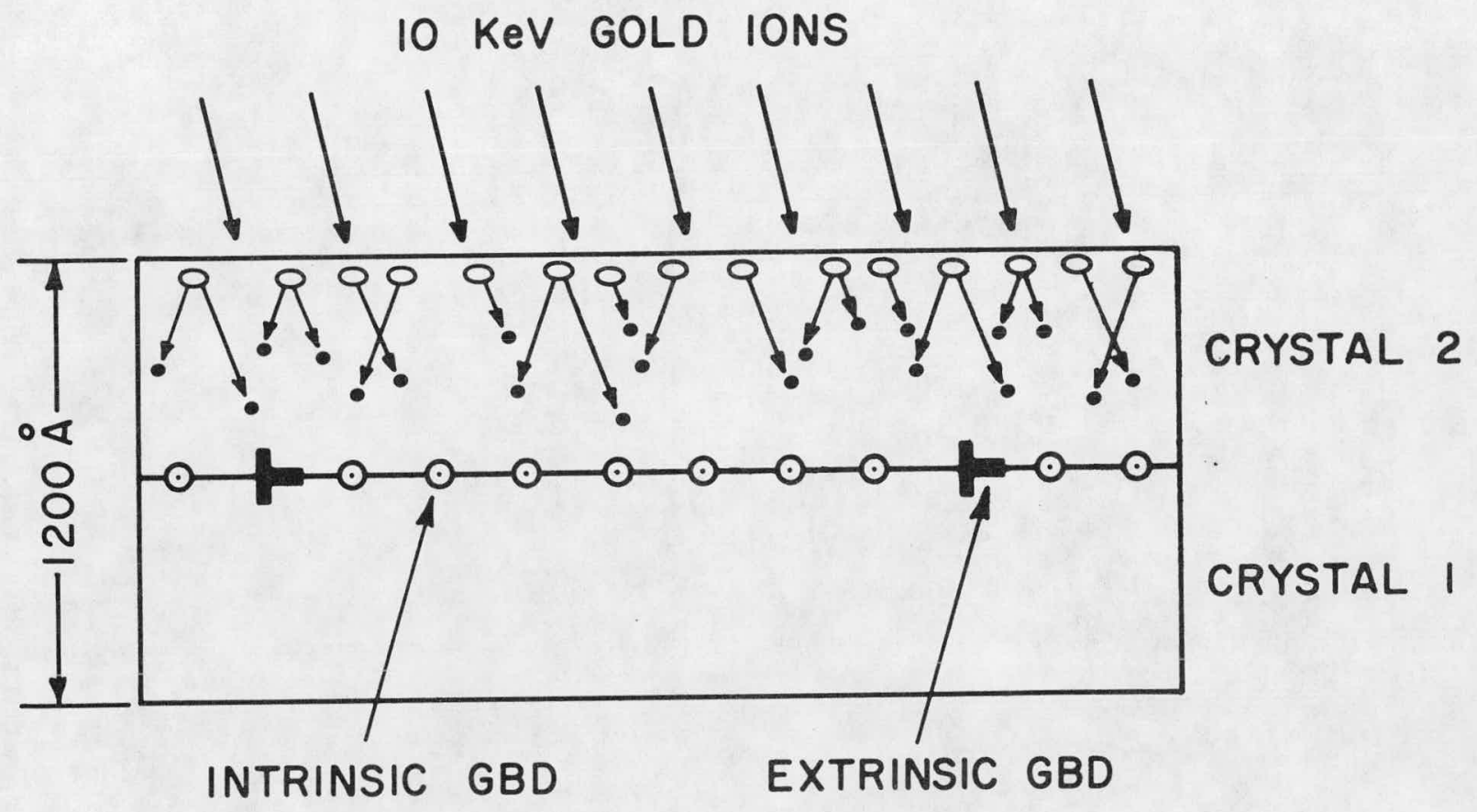

Figure 5. 

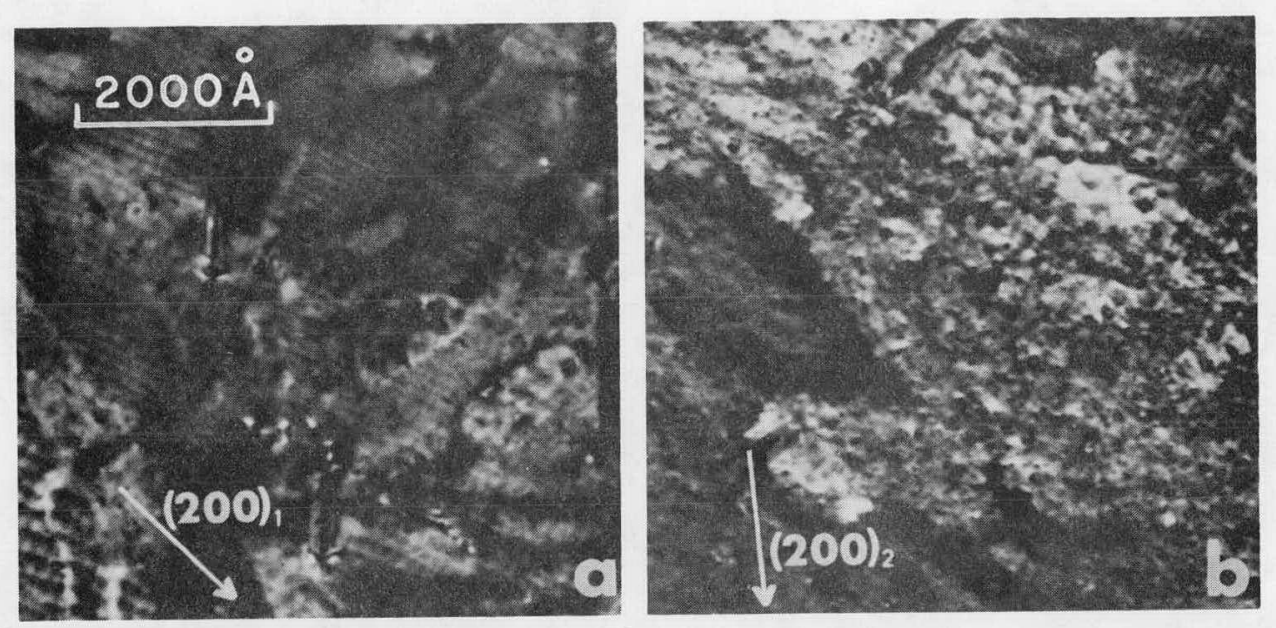

Figure 6 . 


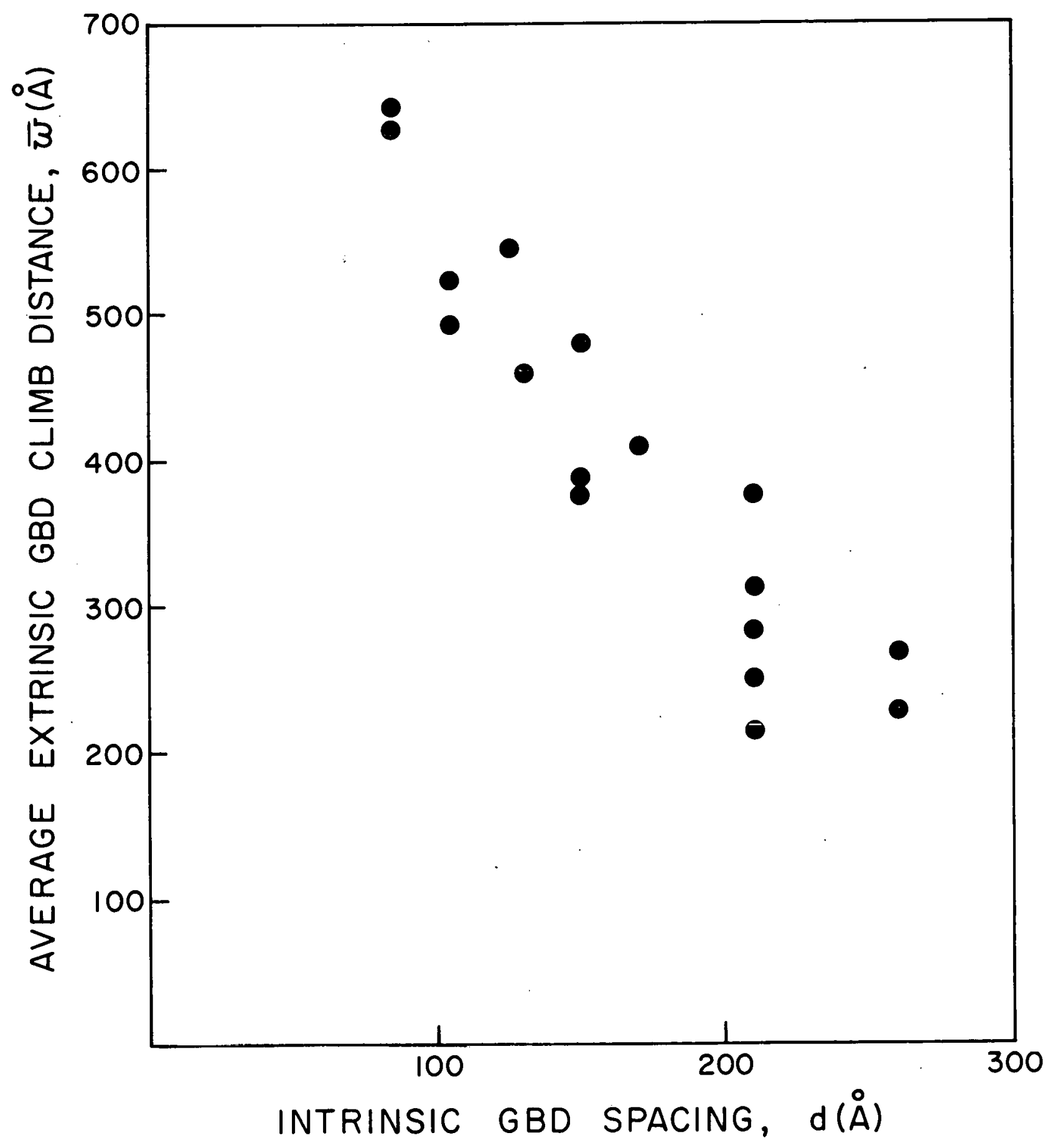

Figure 7 . 